\title{
Síntese de biodiesel a partir da mistura de óleo de soja:gordura animal e óleo fusel
}

\author{
Celeri, E. P. ${ }^{1 *}$; Barañano, A. G. ${ }^{2}$ \\ 1 Curso de Física, Universidade Federal do Espírito Santo, Alegre, ES, Brasil. \\ 2 Departamento de Engenharia Rural, Universidade Federal do Espírito Santo, Alegre, ES, Brasil.
}

*eliomar.pivante@gmail.com

\section{Resumo}

Biodiesel é um biocombustível, obtido através da reação de transesterificação, promissor substituto do diesel de petróleo. Os benefícios de sua utilização é que a combustão deste biocombustível emite menores quantidades de gases poluentes e por ser renovável. A utilização do biodiesel não é amplamente empregada devido ao seu valor comercial ser superior ao do diesel. Uma das formas de tornar o biodiesel economicamente competitivo com o diesel é utilizando matérias-primas de menor valor para transesterificação. Neste trabalho, foi proposto a utilização de misturas óleo de soja:gordura suína para síntese de biodiesel. A gordura suína é uma fonte de triglicerídeo mais econômica, substituir parte do óleo de soja por esta matéria-prima é uma forma de reduzir o custo de produção, outra alternativa empregada foi a utilização do óleo fusel, um rejeito da produção de etanol, como fonte alcoólica. A transesterificação ocorreu em batelada durante 1 hora utilizando uma proporção álcool: mistura óleo de soja e gordura suína de 9:1 e KOH como catalisador. Os resultados da viscosidade cinemática a $40^{\circ} \mathrm{C}$, massa específica a $20^{\circ} \mathrm{C}$ e índice de acidez encontram-se dentro dos limites da ANP evidenciando a possibilidade de utilização destes materiais para produção de biodiesel.

\begin{abstract}
Biodiesel is a biofuel, obtained through the transesterification reaction, promising substitute for petroleum diesel. The benefits of using it are that the combustion of this biofuel emits fewer amounts of pollutants and is renewable. The use of biodiesel is not widely used due to its commercial value is higher than diesel. One of the ways to make biodiesel economically competitive with diesel is to use lower-cost feedstocks for transesterification. In this work, it was proposed the use of mixtures soybean oil: pork fat for biodiesel synthesis. The swine fat is a cheaper source of triglyceride, replacing part of the soybean oil with this raw material is a way to lower the cost of production, another alternative used was the use of fusel oil, a waste from the production of ethanol, as alcoholic source. Transesterification was carried out in a batch for 1 hour using an alcohol:soybean oil and swine fat 9:1 and $\mathrm{KOH}$ as the catalyst mixture. The results of kinematic viscosity at $40^{\circ} \mathrm{C}$ specific mass at $20^{\circ} \mathrm{C}$ and acidity index are within the limits of the ANP showing the possibility of using these materials for biodiesel production.
\end{abstract}

Palavras chaves: Transesterificação, Biodiesel, Óleo Fusel, Gordura Suína, Óleo de Soja

\section{Introdução}

Biodiesel é um biocombustível renovável obtido através da reação de transesterificação de um óleo ou gordura com um álcool de cadeia curta na presença de um catalisador [1] [2].

Transesterificação é o nome dado a reação orgânica em que um éster é transformado em outro através da substituição do grupo alcóxido [3].

Ao ser submetida à transesterificação, a matéria-prima (triglicerídeo) reage e passa a ter características físico químicas semelhantes a do diesel de petróleo podendo ser utilizada em motores diesel sem causar dano [4].
A combustão do biodiesel emite menores quantidades de gases como óxido de enxofre, monóxido e dióxido de carbono e hidrocarbonetos aromáticos quando comparados a combustão do diesel puro [4].

Por lei é obrigatório a adição de $10 \%$ de biodiesel no diesel comercializado em território nacional, essa mistura é chamada B10. O empecilho para utilização do biodiesel puro parte do fato do preço do biodiesel ser superior ao diesel convencional. Geralmente os materiais utilizados para síntese do biodiesel são de interesse no consumo humano fazendo assim com que agregue valor elevando o preço final do produto [5]. 


\section{Blucher Proceedings \\ IX Encontro Científico de Física \\ Aplicada}

Blucher

Gorduras animais são economicamente viáveis para produção de biodiesel por serem uma fonte de triglicerídeos de menor valor comparado a óleos vegetais, contudo, por se tratar de gorduras com altas quantidades de ácidos graxos saturados fazem com que o biodiesel se torne mais viscoso, geralmente acima dos limites para comércio que é de $6 \mathrm{~mm}^{2} / \mathrm{s}$, o que leva esta matéria prima ser pouco utilizada [6].

Com a proposta de utilizar materiais com baixo valor, [5] propôs a utilização de mistura binária de óleo de soja:gordura suína com óleo fusel para obtenção de ésteres alquílicos. Desta forma, substituiria parte do óleo de soja por uma fonte de triglicerídeo mais econômica e usaria, como fonte alcoólica, o óleo fusel. Os resultados de [5] apontam que a viscosidade cinemática a $40^{\circ} \mathrm{C}$ e massa específica a $20^{\circ} \mathrm{C}$ do biodiesel experimental estão acima dos valores estipulados pela ANP. A metodologia utilizada por [5] para a síntese do biocombustível não é amplamente explorada na literatura, que é a transesterificação em duas etapas, assim como o catalisador, solução de metilato de sódio na concentração de $1 \% \mathrm{~m} / \mathrm{m}$, que não é de comum uso para a produção de biodiesel.

Neste trabalho propomos uma alteração metodológica de [5], realizando a reação em uma única etapa, utilizando $\mathrm{KOH}$ como catalisador e a investigação de outras proporções óleo de soja:gordura suína com o objetivo de melhorar os valores de viscosidade cinemática a $40^{\circ} \mathrm{C}$ e massa específica a $20^{\circ} \mathrm{C}$ do produto obtido na reação e assim viabilizar a utilização destas misturas juntamente com óleo fusel tratado para a transesterificação.

\section{Materiais e Métodos}

Para reação do biodiesel experimental foi utilizada uma mistura de $25 \mathrm{~g}$ de óleo de soja:gordura suína nas proporções 0:100, 60:40, 70:30, 80:20, 90:10 e 100:0. A fonte alcoólica foi o óleo fusel tratado na proporção 9:1 e o catalisador empregado foi $\mathrm{o} \mathrm{KOH}, 2 \% \mathrm{em}$ relação a massa da mistura óleo de soja:gordura animal. A reação foi em balão de fundo redondo, imerso em banho maria a $60^{\circ} \mathrm{C}$, com agitação magnética constante, sob refluxo, por $1 \mathrm{~h}$.
Após o processo reacional, o biodiesel foi levado a um funil de decantação e adicionado $50 \mathrm{~mL}$ de uma solução aquosa de HCL (0,5\% v:v) para neutralização, decantação e retirada da glicerina (fase inferior). A fase superior foi lavada 3 vezes com $50 \mathrm{~mL}$ de água deionizada, seguindo, o biodiesel foi colocado em chapa aquecedora a $105^{\circ} \mathrm{C}$ durante $2 \mathrm{~h}$. A umidade foi medida em uma balança de umidade, marca Shimadzu modelo MOC63u, e quando as amostras apresentavam teor de umidade inferior a 1\%, estas eram levadas para caracterização (índice de acidez, massa específica a $20^{\circ} \mathrm{C}$, viscosidade cinemática a $40^{\circ} \mathrm{C}$ e o rendimento). A ocorrência da reação foi verificada por cromatografia em camada delgada, sendo o eluente, mistura de éter de petróleo, éter etílico e ácido acético na proporção (80:16:4), a revelação da placa foi feita em uma cuba contendo cristais de iodo.

Para analisar o índice de acidez foi pesado $2,5 \mathrm{~g}$ de biodiesel e solubilizado em uma mistura de $50 \mathrm{~mL}$ de éter etílico com álcool etílico (2:1 v/v). Acrescentou-se 3 gotas de fenolftaleína 1\% e titulou-se com uma solução de $\mathrm{NaOH} 0,1 \mathrm{~N}$, o volume gasto da solução de $\mathrm{NaOH}$ foi anotado e calculou-se como em [7].

A massa específica $20^{\circ} \mathrm{C}$ foi medida em um densímetro digital modelo DMA 4500 da marca Anton Paar.

Para o cálculo da viscosidade cinemática a $40^{\circ} \mathrm{C}$ utilizou-se a metodologia de [7].

O rendimento foi analisado a partir da quantidade de ácidos graxos convertidos em ésteres alquílicos, utilizando-se a massa final de biodiesel dividida pela massa da mistura óleo de soja:gordura animal alimentada ao reator multiplicado por 100 .

\section{Resultados e Discussão}

A partir da cromatografia, percebeu-se que apesar da ocorrência da conversão de triglicerídeos em biodiesel em todas as amostras, verificam-se mono e diglicerídeos não reagidos, Figura 1.

A partir da caracterização físico química, Tabela 1, pode-se verificar que todas as amostras do biodiesel experimental se encontram dentro do limite estipulado pela ANP, diferente de [5] onde tanto o valor da massa específica, quanto a viscosidade estavam acima. 


\section{Encontro Científico de Física Aplicada}

O rendimento de todas as amostras foi superior a 50\%, Tabela 2. Este resultado já era esperado devido a placa de cromatografia em camada delgada apresentar tri, di e monoglicerídeos não reagidos.

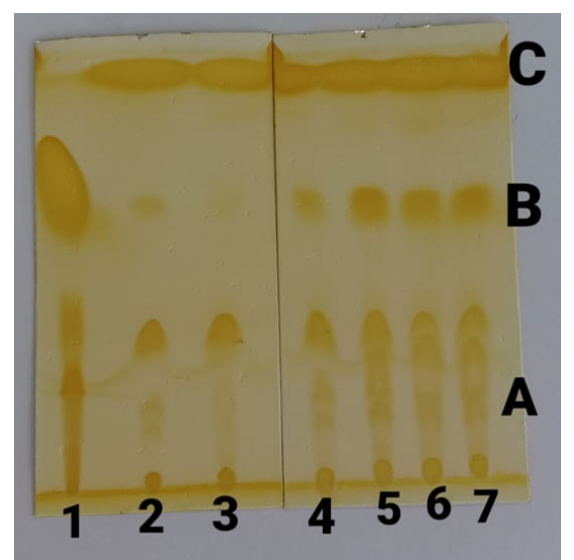

Figura 1: Placa cromatográfica em camada delgada. 1 triglicerídeo, 2 mistura 0:100, 3 mistura 60:30, 4 mistura 70:30, 5 mistura 80:20, 6 mistura 90:10, 7 mistura 100:0, A região de mono e diglicerídeos, B região de triglicerídeos e $\mathbf{C}$ região do biodiesel experimental

Tabela 1: Caracterização físico-química

\begin{tabular}{|c|c|c|c|}
\hline Mistura & $\begin{array}{c}\text { Índice de acidez } \\
\mathrm{mg}(\mathrm{KOH}) / \mathrm{g}\end{array}$ & $\begin{array}{l}\text { Viscosidade } \\
\text { Cinemática a } \\
40^{\circ} \mathrm{C}\left(\mathrm{mm}^{2} / \mathrm{s}\right)\end{array}$ & $\begin{array}{c}\text { Massa } \\
\text { específica a } \\
20^{\circ} \mathrm{C}\left(\mathrm{g} / \mathrm{cm}^{3}\right)\end{array}$ \\
\hline 0:100 & $\begin{array}{c}0,46 \\
( \pm 0,02)\end{array}$ & 4,77 & $\begin{array}{c}0,8788 \\
( \pm 0,002)\end{array}$ \\
\hline $60: 40$ & $\begin{array}{c}0,39 \\
( \pm 0,02)\end{array}$ & 3,22 & $\begin{array}{c}0,8706 \\
( \pm 0,003)\end{array}$ \\
\hline $70: 30$ & $\begin{array}{c}0,43 \\
( \pm 0,01)\end{array}$ & 4,36 & $\begin{array}{c}0,8824 \\
( \pm 0,001) \\
\end{array}$ \\
\hline $80: 20$ & $\begin{array}{c}0,48 \\
( \pm 0,02)\end{array}$ & 4,43 & $\begin{array}{c}0,8871 \\
( \pm 0,001)\end{array}$ \\
\hline 90:10 & $\begin{array}{c}0,46 \\
( \pm 0,01)\end{array}$ & 3,87 & $\begin{array}{c}0,8812 \\
( \pm 0,002)\end{array}$ \\
\hline 100:0 & $\begin{array}{c}0,41 \\
( \pm 0,005) \\
\end{array}$ & 4,61 & $\begin{array}{c}0,8810 \\
( \pm 0,002) \\
\end{array}$ \\
\hline [5] & 0,07 & 6,5 & 0,91 \\
\hline ANP & Até 0,5 & $3-6$ & $0,85-0,90$ \\
\hline
\end{tabular}

Tabela 2: Rendimento do biodiesel experimental

\begin{tabular}{cc}
\hline Misturas & Rendimento (\%) \\
\hline $0: 100$ & 57,6 \\
\hline $60: 40$ & 77,8 \\
\hline $70: 30$ & 64,9 \\
\hline $80: 20$ & 79,1 \\
\hline $90: 10$ & 55,4 \\
\hline $100: 0$ & 60,1 \\
\hline
\end{tabular}

\section{Conclusões}

A proposta de utilizar misturas óleo de soja:gordura animal, para produção de biodiesel, utilizando óleo fusel tratado como fonte alcoólica, mostra-se como

\section{Blucher}

uma promissora forma de utilizar materiais com menor valor agregado para a produção de biodiesel. Apesar de haver triglicerídeos, mono e diglicerídeos não reagidos que leva a um rendimento inferior, melhores resultados quanto à viscosidade e massa específica foram encontrados quando comparados a [5], evidenciando assim que a alteração metodológica empregada neste trabalho foi eficaz.

A utilização de óleo fusel e misturas óleo de soja:gordura animal para produção de biodiesel não é explorada na literatura, sendo assim necessário mais estudos utilizando estes materiais na produção de biodiesel para que um melhor rendimento possa ser alcançado.

\section{Referências}

[1] DEFANTI, L. S. et al. Revista de Divulgação do projeto Universidade Petrobras e If Fluminense. v.1. p. 11-21, 2010

[2] NUNES, J. C. et al. IN: VIII Congresso Brasileiro de Engenharia Química em Iniciação Científica. 2009

[3] GARCIA, C. M. Transesterificação de óleos vegetais. Dissertação (Mestrado em Química), Programa de pós graduação em Química, Instituto de Química, Universidade Estadual de Campinas, Campinas, 2006

[4] DAPIEVE, D. R. Análise da influência da temperatura sobre propriedades físico-químicas de amostras de diesel, biodiesel e suas misturas. Dissertação (Mestrado em Tecnologias Ambientais) Programa de Pós-Graduação em Tecnologias Ambientais. Universidade Tecnológica Federal do Paraná, Medianeira, 2015.

[5] PEREIRA, A. F. Estudo da transesterificação de óleo de soja e gordura suína com óleo fúsel. Tese (Doutorado em Ciências e Tecnologia de Alimentos) Programa de Pós-Graduação em Ciência e Tecnologia de Alimentos. Universidade Federal de Viçosa, Viçosa, 2011.

[6] CUNHA, M. E. da. Caracterização de biodiesel produzido com misturas binárias de sebo bovino, óleo de frango e óleo de soja. Dissertação (Mestrado em Química) Programa de Pós-Graduação em Química, Instituto de química, Universidade Federal do Rio Grande do Sul, Porto Alegre, 2008.

[7] MILLI, B. B. et al ENCICLOPÉDIA BIOSFERA, Centro Científico Conhecer. v.7, n.12, p1-26, 2011. 